\title{
Propofol administration to the fetal-maternal unit preserved cardiac function in late-preterm lambs subjected to severe prenatal asphyxia and cardiac arrest
}

\author{
Matthias Seehase ${ }^{1,2^{*}}$, Patrick Houthuizen ${ }^{3}$, Reint K Jellema ${ }^{2}$, Jennifer JP Collins ${ }^{2}$, Otto Bekers ${ }^{4}$, Boris W Kramer ${ }^{2}$ \\ From 50th Workshop for Pediatric Research \\ Gottingen, Germany. 20-21 March 2014
}

\begin{abstract}
Aims
Cardiac dysfunction is reported after severe perinatal asphyxia. We hypothesized that maternal propofol anaesthesia during emergency caesarean section diminished cardiac injury in preterm fetuses exposed to global severe asphyxia in utero in comparison to isoflurane anesthesia. We tested if propofol decreased the activity of pro-apoptotic caspase-3 by activating the anti-apoptotic AKT kinase family and the signal transducer and activator of transcription-3 (STAT-3).
\end{abstract}

\section{Methods}

44 late-preterm lambs underwent standardized umbilical cord occlusion (UCO) or sham-treatment in utero. UCO resulted in global asphyxia and cardiac arrest. Mothers were randomized to either propofol or isoflurane anaesthesia. After emergency caesarean section, the fetuses were resuscitated and anaesthetized for $8 \mathrm{~h}$ by the anaesthesia of their mothers.

\section{Results}

Propofol treatment resulted $8 \mathrm{~h}$ after UCO in reduced troponin $\mathrm{T}$ levels, in a higher median left ventricular ejection fraction of $84 \%$ in comparison to isoflurane $(74 \%)$, and in reduced activation of caspase-3. Phosphorylated STAT-3 and AKT kinase family were increased to $655 \%$ and $500 \%$ with propofol after asphyxia.

\section{Conclusions}

Propofol administration preserved cardiac function of late-preterm lambs after asphyxia better than isoflurane. The underlying mechanism may be an activation of the anti-apoptotic STAT-3 and AKT pathway.

\section{Authors' details}

'Department for Pediatric Cardiology and Intensive Care Medicine, GeorgAugust-University Göttingen, Germany. ${ }^{2}$ Dept. of Pediatrics, Maastricht University Medical Center, Maastricht, The Netherlands. ${ }^{3}$ Department of Physiology, Cardiovascular Research Institute Maastricht (CARIM), Maastricht University Medical Center, Maastricht, The Netherlands. ${ }^{4}$ Department of Clinical Chemistry, Maastricht University Medical Center, Maastricht, The Netherlands.

Published: 11 September 2014

doi:10.1186/2194-7791-1-S1-A1

Cite this article as: Seehase et al:: Propofol administration to the fetalmaternal unit preserved cardiac function in late-preterm lambs subjected to severe prenatal asphyxia and cardiac arrest. Molecular and Cellular Pediatrics 2014 1(Suppl 1):A1. 